\title{
High Mobility Stemless InSb Nanowires
}

\author{
Ghada Badawy $^{1}$, Sasa Gazibegovic ${ }^{1,2}$, Francesco Borsoi ${ }^{2}$, Sebastian Heedt $^{2}$, Chien-An Wang ${ }^{2}$, \\ Sebastian Koelling $^{2}$, Marcel A. Verheijen ${ }^{1,3}$, Leo P. Kouwenhoven ${ }^{2,4}$ and E. P. A. M. Bakkers ${ }^{1,2, *}$ \\ ${ }^{1}$ Department of Applied Physics, Eindhoven University of Technology, 5600 MB \\ Eindhoven, The Netherlands \\ ${ }^{2}$ QuTech and Kavli Institute of NanoScience, Delft University of Technology, 2600 GA \\ Delft, The Netherlands \\ ${ }^{3}$ Eurofins Material Science Netherlands B.V., High Tech Campus 11, 5656 AE Eindhoven, \\ The Netherlands \\ ${ }^{4}$ Microsoft Quantum Lab Delft, 2600 GA Delft, The Netherlands \\ *Corresponding Author Email: e.p.a.m.bakkers@tue.nl.
}

\section{Supporting Information}

\section{S1 Substrate Fabrication}

In what follows we describe the steps to fabricate the substrates used in this work.

\section{Selective-Area Mask Deposition}

- Start out with a 2-inch indium-antimonide (111)B wafer.

- The wafer as taken from the supplier box, is put in the plasma-enhanced chemical vapor deposition (PECVD) chamber, where around $20 \mathrm{~nm}$ of silicon nitride $\left(\mathrm{SiN}_{\mathrm{x}}\right)$ are deposited.

\section{Polymer Resist for Electron-Beam Lithography}

- A primer (AR300-80) is spin coated on the sample at $4000 \mathrm{rpm}$ for 60 seconds.

- The primer is baked on a hotplate for 2 minutes at $180^{\circ} \mathrm{C}$.

- An electron beam resist (AR-P 6200.04) is spin coated on the sample at 4000 rpm for 60 seconds.

- The resist is baked on a hotplate for 3 minutes at $150^{\circ} \mathrm{C}$.

- The sample is loaded to an electron beam lithography (EBL) tool where the design is written. The design consists of fields of nano-dots of varying sizes $(16 \mathrm{~nm}-50 \mathrm{~nm})$ and varying inter-dot spacing (pitch) ranging from $0.25 \mu \mathrm{m}$ to $4 \mu \mathrm{m}$.

\section{Resist Development}

- The sample is then taken out of the EBL tool for development. The sample is developed in Developer AR 600-546 for 60 seconds. It is then dipped in a beaker containing a Stopper AR 600-60 for 30 seconds. The sample is then rinsed in isopropanol (IPA) for 1 minute.

- The sample is dried using a nitrogen gun. 


\section{Etching of $\operatorname{SiN}_{x}$ Through Resist Nano-holes}

- Wet etching: the sample is dipped in a beaker containing buffered oxide etch BOE 7:1 $\left(\mathrm{HF}: \mathrm{NH}_{4} \mathrm{~F}\right)$ for 14 - 25 seconds depending on the desired mask opening size. After that, the sample is rinsed in running ultra pure water (UPW) for at least 4 minutes.

- The sample is dried using a nitrogen gun.

\section{Gold Evaporation}

- The samples are loaded in an electron-beam evaporator, where $8 \mathrm{~nm}$ of gold evaporated onto the sample with a deposition rate of $1 \AA$ /second. Thereby gold is deposited within the holes in the $\mathrm{SiN}_{\mathrm{x}}$ mask as well as everywhere on the resist.

\section{Lift-off}

- The sample is dipped into PRS3000 (a photoresist stripper) for 30 minutes where a stir magnet continuously stirs the liquid.

- The sample is then transferred to an acetone beaker. There it is placed in a mildly sonicated bath for 5 minutes to ensure proper removal of gold residues.

- The sample is finally rinsed in an isopropanol beaker for 2 minutes.

- A nitrogen gun is used to dry the sample.

\section{Oxygen Plasma}

- The sample is then exposed to 300 Watt oxygen plasma with a gas flow of 55 standard cubic $\mathrm{cm}$ (sccm) for 2 minutes to remove any organic residues and ensure sample cleanliness.

\section{S2 Growth Details}

Growth in this work was done using a horizontal Aixtron 200 metal-organic vapor phase epitaxy (MOVPE) reactor. The samples were heated to the desired growth temperature of $495^{\circ} \mathrm{C}$ by infrared lamps. The used precursors were trimethyl-indium (TMIn) and trimethyl-antimony (TMSb), where the TMIn molar fraction was kept constant at $1.95 \times 10^{-7}$ and the TMSb was varied from $6.17 \times 10^{-5}$ to $1.41 \times 10^{-3}$. The samples were heated up and cooled down under a flow of TMSb. The growth time was varied from 5 minutes to 6 hours. The carrier gas was $\mathrm{H}_{2}$ and during growth the reactor pressure was kept constant at 50 mbar and a total volume of 6000 liters. 


\section{S3 Seeding of SA-VLS InSb NWs}

Growth of SA-VLS wires is consistent with selective-area seeding, where nucleation is preferred at edge sites. Therefore we observe the Au particles being consistently pushed to the edge of the mask opening, as shown in Figure S1. Once the opening is completely filled with precipitated InSb, the Au droplet is centered with respect to the mask opening, showing InSb NW diameters larger than the Au diameter.

It has been observed that $\mathrm{Sb}$ exposure has an effect on the morphology of Au particles in the early stages of growth causing nanowires to grow rather in-plane, leaving a streak of material behind, as the gold droplet moves [1]. As shown in Figure S1 a streak of material in the opening of the mask is visible after 5 minutes. As the Au particle encounters the mask edge, it does not like to wet it and hence bounces off (or it is being pushed around) the edges of the mask until the exposed substrate is filled. The resulting growth does not yield well-defined facets, causing the catalyst to reside on the smallest formed "facet", as no stable/defined planes are yet formed, as shown after 15 minutes of growth (see Figure S1). As growth proceeds, well-defined facets are slowly formed, possibly by catalyst wetting of more than one facet, until a (111) B plane is developed causing the gold catalyst to end up on it. Once this happens, normal NW growth takes place, where axial growth is driven by the catalyst whereas non-tapered radial growth is governed by VSS, resulting in slanted (111)A facets towards the catalyst, as shown in Figure S1 (60 minutes), similar to what has been observed for InAsSb wires [2]. This stable wire growth, with fully formed facets, results in hexagonal cross-section NWs, as shown in the inset of Figure S1.
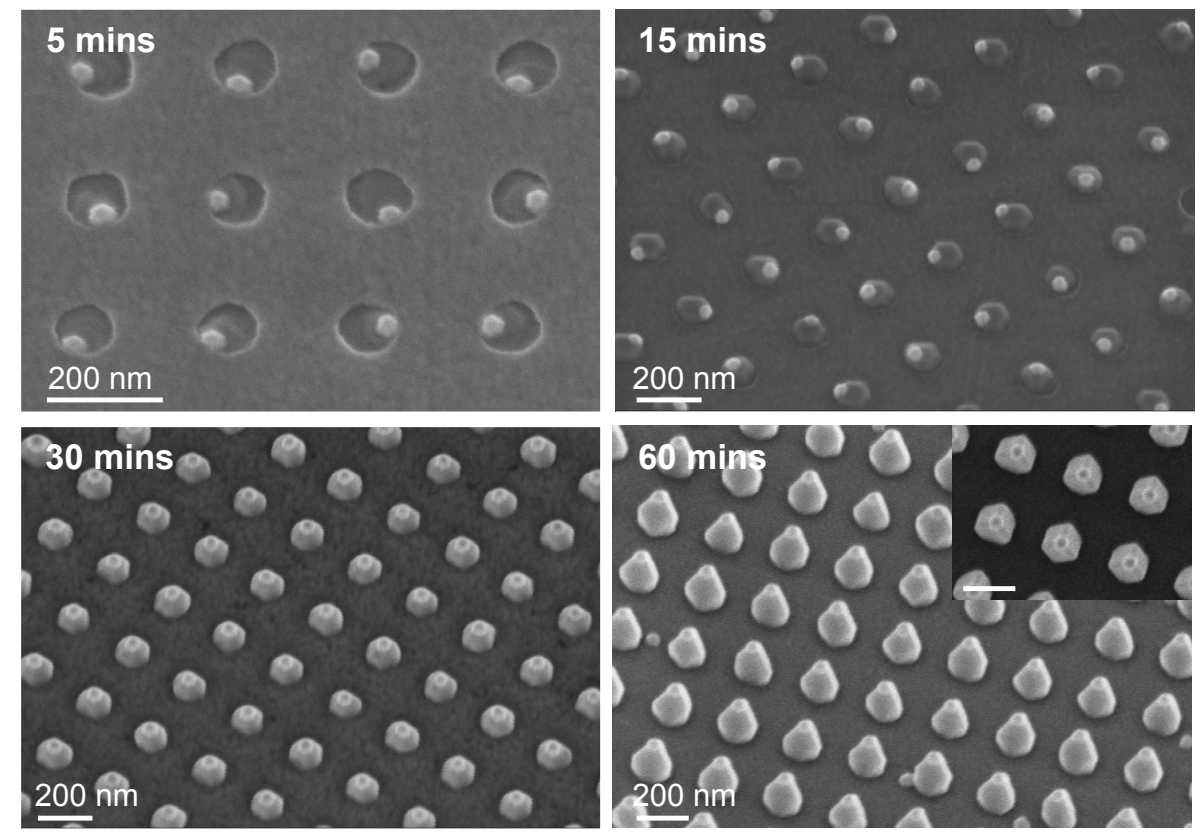

Figure S1: 30-degree tilt SEM images showing different growth stages of InSb NWs from 5 to 60 minutes of growth. The growth is performed for samples with the biggest examined openings in the $\mathrm{SiN}_{\mathrm{x}}$ mask, around $130 \mathrm{~nm}$ openings. The gold droplet is approximately $30 \mathrm{~nm}$ in diameter prior to growth. Starting half an hour of growth, the gold droplet seems to take the stable configuration of being positioned on top of the growing wires. After 60 minutes of growth, stable facets and a hexagonal cross-section is discernable in the top-view inset.

The yield of these wires is sometimes limited by gold dots sliding under the mask during early growth stages, as indicated in Figure S2. The sliding is confirmed by closer examination yielding a brighter spot next to the holes with missing gold. This is observed for bigger inter-opening spacing (cf. with Figure S2). 


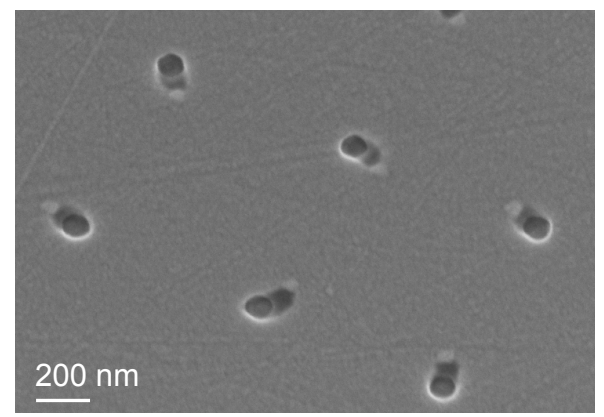

Figure S2: 30-degree tilt SEM image Au dots sliding under the selective-area mask, indicated by a bright dot next to the opening and empty holes. The image corresponds to 5 minutes of growth.

\section{S4 Volume Effect}

The volume effect refers to the fact, that if two objects have to grow to the same volume, the one with the smaller width (diameter) will grow higher, i.e. exhibit a higher aspect-ratio. The schematic in Figure S3 serves as a visualization of the volume effect.
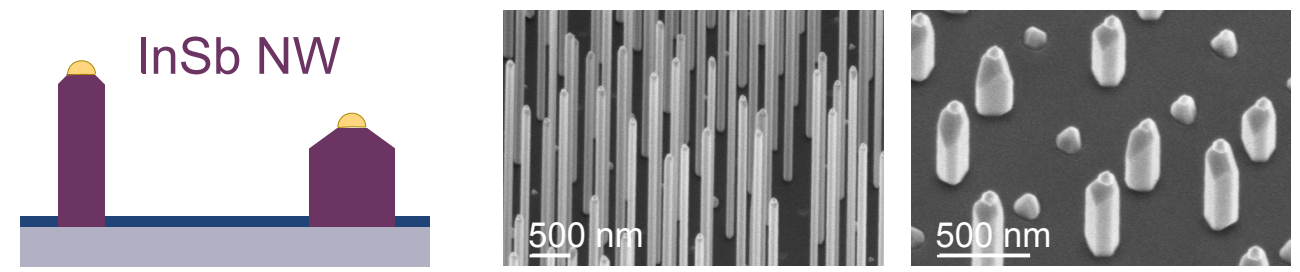

Figure S3: Visualization of the volume effect, reflecting lower (higher) axial growth rates for bigger (smaller) diameter wires for a given volume. 


\section{S5 TMSb inhibiting parasitic growth}

The dependence of parasitic growth on pre-growth conditions. As shown in Figure S4, depending on the heat-up flow and the TMIn concentration prior to growth, parasitic growth is either favoured or suppressed. All the experiments shown in Figure S4 have the same growth parameters, they only differ in heat-up flow and TMIn filling of the gold droplet before growth commences. For the case where the gold catalyst is not filled with TMIn, we observe that parasitic growth decreases with increasing concentration of TMSb flow during heat-up. This observation indicates that parasitic growth is initiated by In deposition on the mask. TMIn diffusion is thus enhanced in presence of high enough TMSb concentration. Additionally, for the highest TMSb heat-up flow, parasitic growth is favoured as the TMIn filling duration increases. Thus indicating, that for a high enough TMIn concentration, nucleation on the mask becomes more probable.

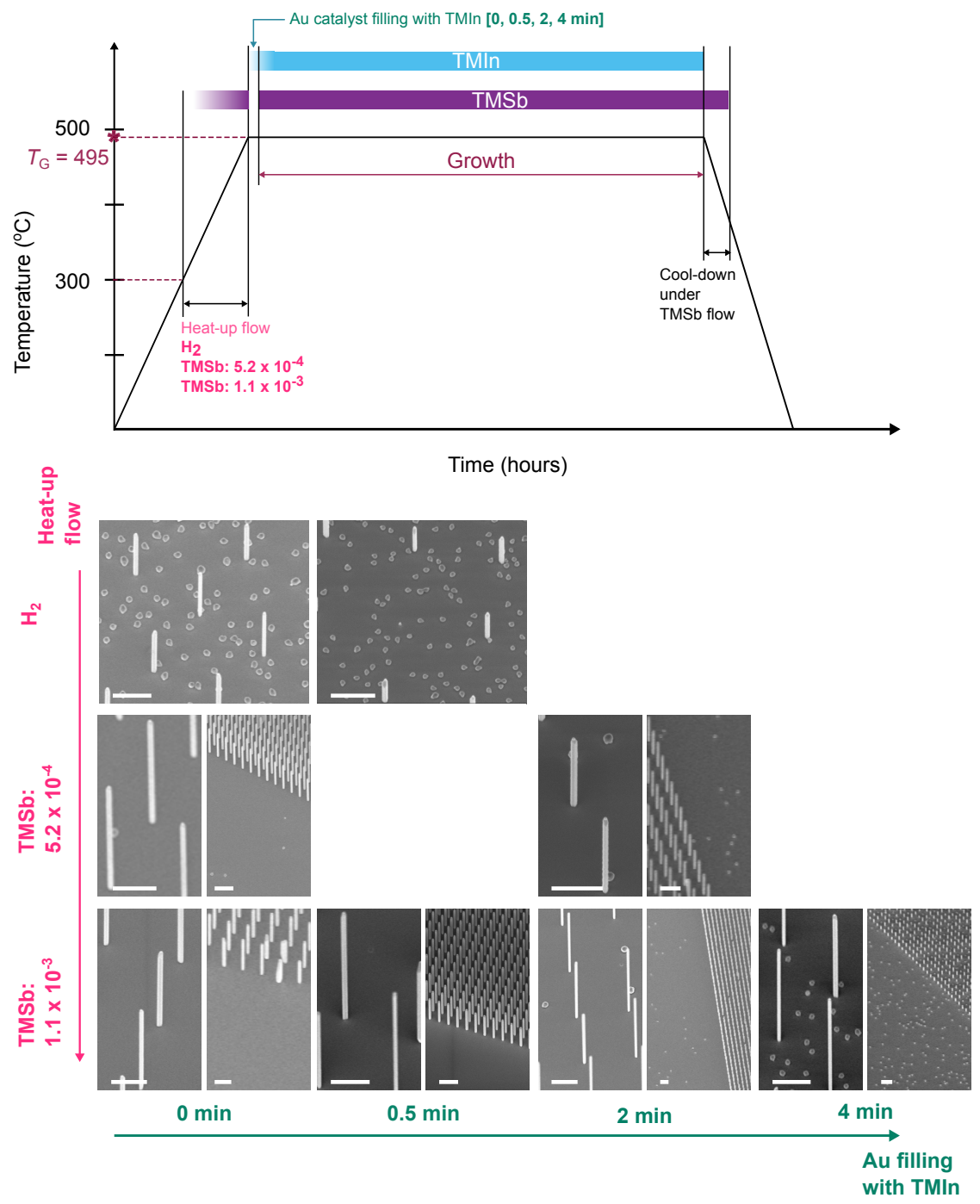

Figure S4: Top panel showing the growth scheme used to grow stemless InSb NWs. Temperature is ramped up from room temperature to the growth temperature $\left(T_{\mathrm{G}}\right)$ of $495{ }^{\circ} \mathrm{C}$. In a few experiments (as shown in the bottom panel), the sample goes from $300{ }^{\circ} \mathrm{C}$ to $495{ }^{\circ} \mathrm{C}$ under a flow of TMSb. Once $T_{\mathrm{G}}$ is reached, the TMSb flow is switched off and the TMIn is opened for a short period of time to fill the gold catalyst with TMIn. The filling time is varied from 0 seconds and up to 4 minutes for different experiments, as shown in the bottom panel. As the Au filling is completed, the TMSb is switched on again, thus commencing growth. After growth is completed, the sample is cooled down from $T_{\mathrm{G}}$ to $370{ }^{\circ} \mathrm{C}$ under a flow of $\mathrm{TMSb}=1.1 \times 10^{-3}$. The bottom panel shows representative SEM images from different experiments where different combinations of heat-up flow and Au filling durations are varied, while growth parameters are identical. Scale bar corresponds to $1 \mu \mathrm{m}$. 


\section{S6 Pitch Dependence}

Dependence of NW dimensions on pitch and extraction of surface diffusion length are discussed here. The data are collected from a growth run with low TMSb flow (molar fraction: $8.2 \times 10^{-5}$ ). Observing NW volume as a function of pitch (see Figure S5a), an increase in volume is barely discernible at a pitch of $2 \mu \mathrm{m}$. Thus, we can extract a diffusion length of about $2 \mu \mathrm{m}$. The plot of Figure S5b shows that the NW diameter is more or less independent of pitch, whereas the NW height is pitch-dependent. More generally, the NW height slowly saturates with pitch.
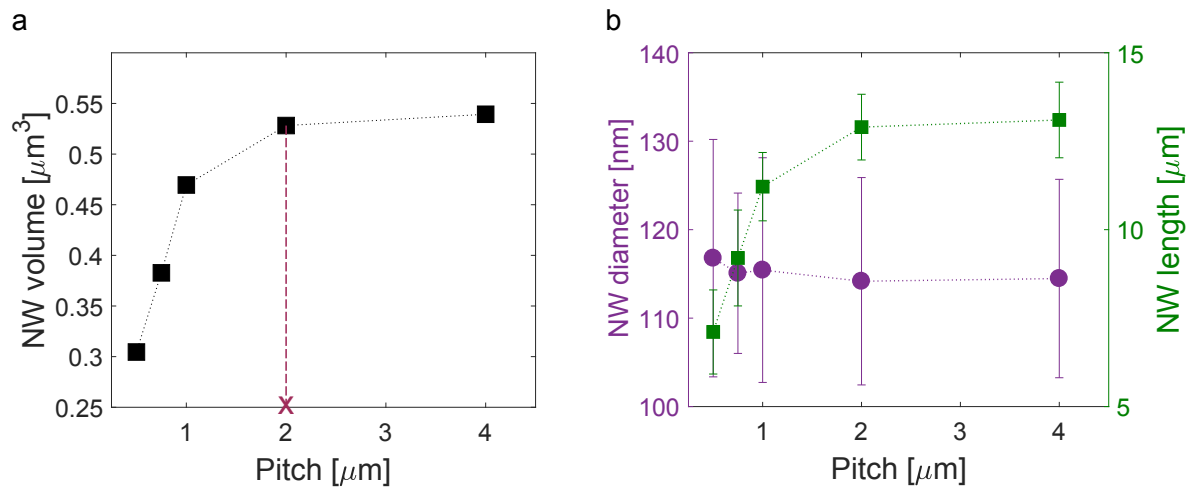

Figure S5: Plots showing dependence of NW dimensions on pitch. (a) NW volume increases with pitch up until an inter-wire spacing of $2 \mu \mathrm{m}$. At this pitch, the NW volume saturates where a surface diffusion length is concluded. (b) Plot of NW diameter and length against pitch. NW diameter is almost pitch-independent as opposed to NW length.

\section{S7 TEM Analysis}

Transmission electron microscopy (TEM) images were taken of the NWs to characterize their crystal quality. Stemless InSb NWs show pure zinc blende atomic ordering.
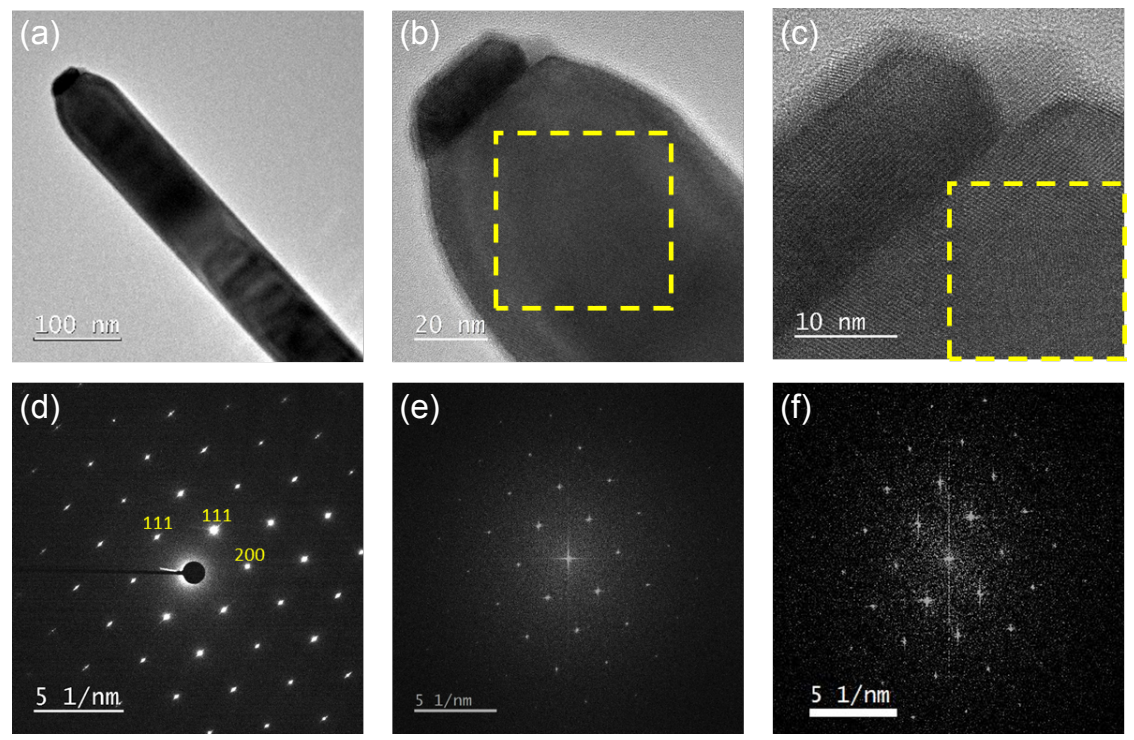

Figure S6: (a) Bright-field TEM image of a stemless InSb NW with a gold catalyst on top. (b), (c) HRTEM images of the top part of this wire. (d) Selected area electron diffraction pattern of the central part of the wire. (e), (f) Fast Fourier Transform patterns of the dashed regions of figures (b) and (c) respectively. All three patterns are characteristic for the $\langle 110\rangle$ zone axis pattern of the zinc blende crystal structure of InSb. 


\section{S8 Device Fabrication}

Here, we describe the fabrication process of both mobility and QPC devices:

- The substrates are made out of $\mathrm{p}^{++}$-doped $\mathrm{Si}$ covered by $285 \mathrm{~nm}$ of thermal $\mathrm{SiO}_{\mathrm{x}}$ via dry oxidation, onto which $\mathrm{Ti} / \mathrm{Au}$ alignment markers are defined. The substrates are cleaned with $10 \mathrm{~min}$ of remote oxygen plasma at a pressure of 2 mbar and a power of $100 \mathrm{~W}$.

- Nanowires are deterministically transferred by means of a nanomanipulator from the growth chip onto the substrate. Optical images are taken in order to facilitate the design of the device with respect to the alignment markers.

- A double layer of resist (PMMA 495K A6 and PMMA 950K A2) is spin-coated on the substrate and each layer is baked for 10 min at $175{ }^{\circ} \mathrm{C}$.

- The pattern of the contacts is defined by electron beam lithography (EBL). Source and drain contacts are designed to be separated by a 'long' InSb channel of length 1 or $2 \mu \mathrm{m}$.

- Development in a solution of MIBK:IPA (1:3) for $1 \mathrm{~min}$, and in IPA for $1 \mathrm{~min}$.

- Prior to the metal deposition, the chip is cleaned with 2 min of remote oxygen plasma at 2 mbar and a power $100 \mathrm{~W}$, and left for $30 \mathrm{~min}$ in a sulphur-rich ammonium polysulfide solution diluted in water with a ratio of $1: 200$ at $60{ }^{\circ} \mathrm{C}$.

- The chip is rinsed thoroughly with water and quickly loaded in an e-gun evaporator. Here it is exposed to $30 \mathrm{~s}$ of helium ion milling at a pressure of $1.6 \times 10^{-2}$ mbar to facilitate the ohmic contact between $\mathrm{InSb}$ and metal, which is then evaporated in the sequence of $10 \mathrm{~nm}$ of $\mathrm{Ti}$, and $170 \mathrm{~nm}$ of $\mathrm{Au}$.

- Lift-off in acetone for 2 hours at $50{ }^{\circ} \mathrm{C}$.

\section{Additional Steps for QPC devices:}

- $40 \mathrm{~nm}$ of $\mathrm{SiN}_{\mathrm{x}}$ gate dielectric is sputtered onto the entire chip.

- Resist (PMMA 495K A4) is spin-coated and baked for $10 \mathrm{~min}$ at $175{ }^{\circ} \mathrm{C}$.

- The pattern of the top gates is defined by EBL; the designed gate widths lie in the range of 120 - $270 \mathrm{~nm}$, comparable to the expected electron mean free path.

- Development in a solution of MIBK:IPA (1:3) for $1 \mathrm{~min}$, and in IPA for $1 \mathrm{~min}$.

- $20 \mathrm{~nm}$ of $\mathrm{Ti}$ and $210 \mathrm{~nm}$ of $\mathrm{Au}$ are deposited in an e-gun evaporator.

- Lift-off in acetone for 2 hours at $50{ }^{\circ} \mathrm{C}$.

Experimental runs 1 and 2 hosting mobility devices (see Table S1) are performed respectively in a dilution refrigerator at a base temperature of $\approx 15 \mathrm{mK}$ and in a dip-stick in helium at $\mathrm{T}=4.2 \mathrm{~K}$. QPC devices are loaded in a He-3 fridge with a base temperature of $300 \mathrm{mK}$. Prior to cool-down, the sample space is pumped for at least 48 hours, similarly to Ref. [3], in order to efficiently desorb adsorbates from the nanowires surface. The chips were imaged via SEM (scanning electron microscopy) only after the measurement to minimize carbon contamination, in order to evaluate diameter and length of the nanowires.

\section{Mobility Measurements}

For the study of the field-effect mobility $\mu$, we accurately reproduce the conditions used in Ref. [3]. NanowireFET devices were fabricated as described in section S8. Based on the channel lengths used, the diffusive long-channel regime is assumed. Hence the current $I$ as a function of back-gate voltage $V_{\mathrm{BG}}$ and dc-bias voltage $V_{\mathrm{dc}}$ can be modeled by 


$$
I\left(V_{\mathrm{BG}}, V_{\mathrm{dc}}\right)=\frac{V_{\mathrm{dc}}}{\left[L^{2} / \mu C\left(V_{\mathrm{BG}}-V_{\mathrm{th}}\right)\right]+R_{\mathrm{c}}}
$$

The saturation current is limited by the series resistance $R_{\mathrm{c}}$, which includes the contact, filter and line resistances. The current pinch-off is reached at the threshold voltage $V_{\mathrm{th}}$. The value of the capacitance is evaluated via a 3D Laplace solver for a typical nanowire device geometry including two metallic leads. In this finite elements model the nanowire is treated as a metal. The capacitance is then reduced by $20 \%$ to include the effect of the quantum confinement. This reduction was in fact found from the comparison of the estimations of the capacitance obtained by a 2D Schrödinger-Poisson solver and by a 2D Laplace solver, as discussed in Ref. [3]. We expect that the inaccuracy in estimating the capacitance due to the unknown surface density of states leads to a relative error of 10-20\% in the mobility - in line with what was evaluated in Ref. [4].

We present here in Table S1 the detailed summary of all devices measured to evaluate the electron mobility in the stemless InSb nanowires. Two chips (which we call experimental runs 1 and 2) have been fabricated and measured. Prior to the cool down, the sample space was evacuated at room temperature for 48 and 65 hours for run 1 and 2, respectively. Onto these chips, nanowires grown from two different growth batches (RB2252 and RB2669) were transferred. We did not observed any significant difference between both evacuation times, and both growth chips. The mobility and threshold voltage values presented in Table S1 are weighted averages of the parameters extracted from the up and down back-gate sweep at $10 \mathrm{mV}$ bias. The error of the mobility reflects the weighted average of the values obtained from the least-square fits of current vs gate voltage traces for the up and down sweeps. The mobility values lie between $3.42 \times 10^{4} \mathrm{Vs} / \mathrm{cm}^{2}$ and $5.7 \times 10^{4} \mathrm{Vs} / \mathrm{cm}^{2}$, with an average value of $4.37 \times 10^{4} \mathrm{Vs} / \mathrm{cm}^{2}$.

Table S1: Summary of FET devices

\begin{tabular}{|c|c|c|c|c|c|c|}
\hline Experimental run & NW batch & Device number & Diameter $(\mathrm{nm})$ & Channel length $(\mu \mathrm{m})$ & $\mathrm{V}_{\text {th }}$ & $\mu \pm \delta \mu$ \\
\hline 1 & RB2252 & 3 & 191 & 1.92 & -1.50 & $44430 \pm 260$ \\
\hline 1 & RB2252 & $5 \mathrm{~b}$ & 168 & 1.9 & -1.13 & $43640 \pm 220$ \\
\hline 1 & RB2252 & 6 & 182 & 1.96 & -1.21 & $42740 \pm 200$ \\
\hline 1 & RB2669 & $14 \mathrm{a}$ & 175 & 1.87 & -1.24 & $41240 \pm 250$ \\
\hline 1 & RB2669 & $14 \mathrm{~b}$ & 169 & 1.89 & -1.40 & $42900 \pm 260$ \\
\hline 1 & RB2669 & $15 \mathrm{a}$ & 159 & 1.94 & -1.20 & $43910 \pm 210$ \\
\hline 1 & RB2669 & $17 \mathrm{a}$ & 171 & 1.92 & -1.06 & $47960 \pm 180$ \\
\hline 1 & RB2669 & $17 \mathrm{~b}$ & 166 & 1.92 & -1.20 & $44150 \pm 220$ \\
\hline 1 & RB2669 & 18 & 165 & 1.87 & -0.99 & $39820 \pm 270$ \\
\hline 1 & RB2669 & 19 & 183 & 1.75 & -1.71 & $40000 \pm 290$ \\
\hline 1 & RB2669 & 20 & 186 & 1.92 & -1.12 & $47760 \pm 450$ \\
\hline 1 & RB2669 & 21 & 189 & 1.93 & -1.21 & $57000 \pm 410$ \\
\hline 2 & RB2252 & 1 & 141 & 1.94 & 0.10 & $41040 \pm 190$ \\
\hline 2 & RB2252 & 2 & 180 & 1.02 & 0.17 & $45590 \pm 150$ \\
\hline 2 & RB2252 & 4 & 138 & 1.95 & 0.45 & $34280 \pm 90$ \\
\hline
\end{tabular}

\section{Additional QPC data}

Here, we present data of a QPC device different from the one reported in the main text. Similarly, the QPC is defined in a nanowire segment by means of a 215 -nm-wide top gate (Figure S7a), while an electric field can be applied by the global back gate across the entire nanowire. The magnetic field is applied perpendicularly to the substrate and breaks the spin degeneracy of the subbands. In case of ballistic transport, this translates in the conductance rising in steps of $e^{2} / h$ versus top-gate voltage. From the Zeeman splitting $\left(E_{\mathrm{Z}}=g \mu_{\mathrm{B}} B\right)$ of the first subband (see Figure S7b), we extracted a value of the g factor of 38, comparable to what was reported in Refs. [5,6]. 
(a)

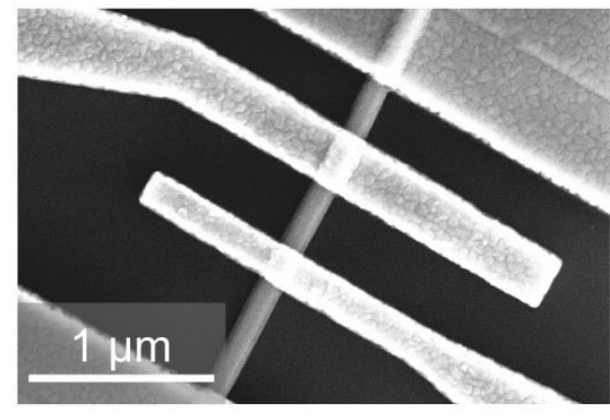

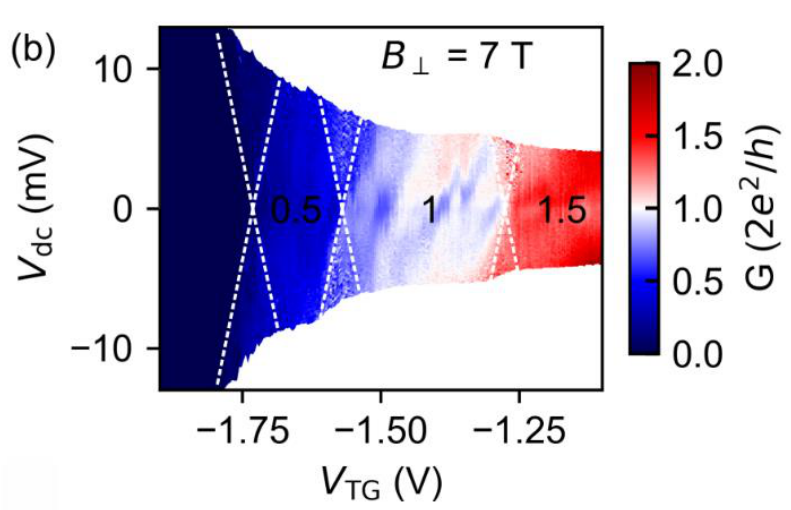

Figure S7: (a) InSb nanowire device comprising two top gates that can be used to electrostatically create QPCs. (b) Conductance as a function of bias voltage $V_{\mathrm{dc}}$ and top-gate voltage $V_{\mathrm{TG}}$ at $B=7 \mathrm{~T}$. The top gate is 215 -nm-wide and the measurement is performed at $T=5 \mathrm{~K}$ with $V_{\mathrm{TG}}=9 \mathrm{~V}$. The bias voltage is corrected for the voltage drop occurring due to additional series resistances.

\section{Additional WAL Data}

Here, the weak antilocalization effect in the magnetoconductance data is modeled using the one-dimensional WAL formula of Kurdak et al. [7] given by

$$
\begin{aligned}
\Delta G(B)= & -\frac{e^{2}}{h} \frac{1}{L}\left[3\left(\frac{1}{l_{\phi}^{2}}+\frac{4}{3 l_{S O}^{2}}+\frac{1}{l_{B}^{2}}\right)^{-1 / 2}-\left(\frac{1}{l_{\phi}^{2}}+\frac{1}{l_{B}^{2}}\right)^{-1 / 2}\right. \\
& \left.-3\left(\frac{1}{l_{\phi}^{2}}+\frac{4}{3 l_{S O}^{2}}+\frac{d}{l_{e}^{2}}+\frac{1}{l_{B}^{2}}\right)^{-1 / 2}+\left(\frac{1}{l_{\phi}^{2}}+\frac{d}{l_{e}^{2}}+\frac{1}{l_{B}^{2}}\right)^{-1 / 2}\right] .
\end{aligned}
$$

The diffusion in the nanowire with the scattering length $l_{\mathrm{e}}$ has the dimensionality $d=3$. Here, $l_{\mathrm{e}}=\nu_{\mathrm{F}} \tau_{\mathrm{e}}$ is extracted from the threshold voltage $V_{\mathrm{th}}$, which yields the Fermi velocity $\nu_{\mathrm{F}}$, and the mobility $\mu$, providing the characteristic scattering time $\tau_{\mathrm{e}}$, that are both determined from FET traces. The magnetic dephasing length $l_{\mathrm{B}}$ and the spin relaxation length $l_{\mathrm{SO}}$ are given by $[8]$

$$
l_{B}=\sqrt{\frac{C_{m}}{d} \frac{l_{m}^{4}}{w_{f}^{\gamma_{m}} l_{e}^{2-\gamma_{m}}}}
$$

and

$$
l_{S O}=\sqrt{\frac{C_{s}}{d} \frac{l_{R}^{4}}{w_{f}^{\gamma_{s}} l_{e}^{2-\gamma_{s}}}},
$$

respectively, with $C_{\mathrm{m}}=22.3, \gamma_{\mathrm{m}}=3.174$ and $C_{\mathrm{s}}=8.7, \gamma_{\mathrm{s}}=3.2$ determined from Monte Carlo simulations of the dephasing along closed-loop electron trajectories in a hexagonal nanowire [8]. Here, $w_{\mathrm{f}}$ refers to the facet-to-facet nanowire diameter and $l_{\mathrm{R}}=\hbar^{2} / m^{*} \alpha_{R}$ is the Rashba spin precession length.

Before fitting the magnetoconductance correction model of eq. 2 to the low-temperature transport data, the conductance background needs to be subtracted. To this end, for every magnetoconductance trace the average conductance at field values $|B|>0.5 \mathrm{~T}$ is subtracted. In Figure S8 the weak antilocalization effect is shown for another device different from the one in the main text, which is depicted in Figure S7a. Here, the magnetoconductance correction is averaged for a back-gate voltage interval from $-1.5 \mathrm{~V}$ to $13.5 \mathrm{~V}$. The orange curve in Figure S8 is the fit of the quasiclassical model in eq. 2, which yields a phase coherence length of $l_{\phi}=510 \mathrm{~nm}$, comparable to the device presented in the main text. The extracted spin-orbit energy is $E_{\mathrm{SO}}=0.37 \mathrm{meV}$. This corresponds to a Rashba parameter of $\alpha_{\mathrm{R}}=0.64 \mathrm{eVA}$. 


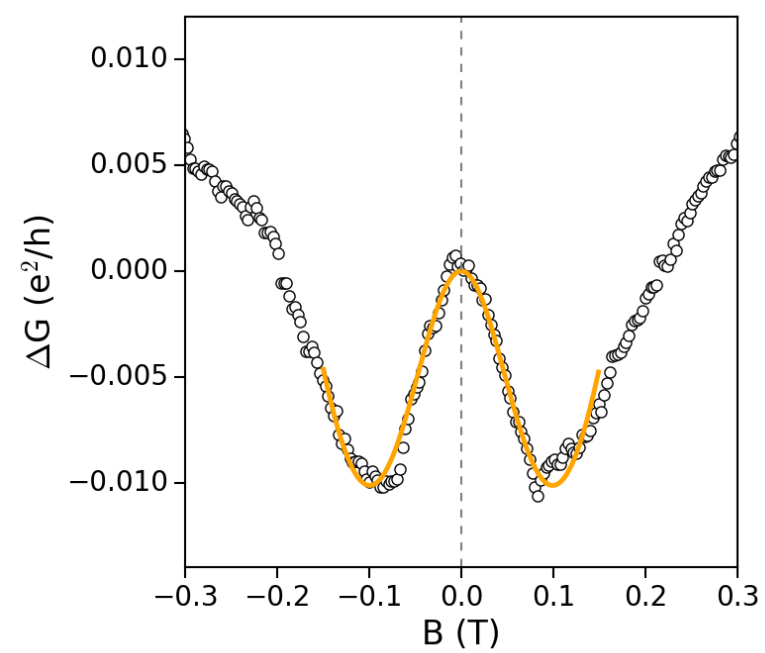

Figure S8: Magnetoconductance correction for the nanowire device depicted in Figure S7a at $T=0.3 \mathrm{~K}$. For the fit (solid line) the quasi-classical WAL model from eq. 2 is utilized [8].

\section{References}

[1] B. Mattias Borg and Lars-Erik Wernersson. Synthesis and properties of antimonide nanowires. Nanotechnology, 24(20):1-18, 2013.

[2] Daniele Ercolani, Mauro Gemmi, Lucia Nasi, Francesca Rossi, Marialilia Pea, Ang Li, Giancarlo Salviati, Fabio Beltram, and Lucia Sorba. Growth of inas/inassb heterostructured nanowires. Nanotechnology, 23(11):115606, 2012.

[3] Önder Gül, David J. Van Woerkom, Ilse van Weperen, Diana Car, Sébastien R. Plissard, Erik P. A. M. Bakkers, and Leo P. Kouwenhoven. Towards high mobility insb nanowire devices. Nanotechnology, 26(21):215202, 2015.

[4] Sebastian Heedt, Isabel Otto, Kamil Sladek, Hilde Hardtdegen, Jürgen Schubert, Natalia Demarina, Hans Lüth, Detlev Grützmacher, and Thomas Schäpers. Resolving ambiguities in nanowire field-effect transistor characterization. Nanoscale, 7(43):18188-18197, 2015.

[5] Henrik A. Nilsson, Philippe Caroff, Claes Thelander, Marcus Larsson, Jakob B. Wagner, Lars-Erik Wernersson, Lars Samuelson, and HQ Xu. Giant, level-dependent g factors in insb nanowire quantum dots. Nano letters, 9(9):3151-3156, 2009.

[6] Jakob Kammhuber, Maja C. Cassidy, Hao Zhang, Önder Gül, Fei Pei, Michiel W. A. de Moor, Bas Nijholt, Kenji Watanabe, Takashi Taniguchi, Diana Car, et al. Conductance quantization at zero magnetic field in insb nanowires. Nano letters, 16(6):3482-3486, 2016.

[7] C. Kurdak, A. M. Chang, A. Chin, and T. Y. Chang. Quantum interference effects and spin-orbit interaction in quasi-one-dimensional wires and rings. Physical Review B, 46(11):6846, 1992.

[8] I. Van Weperen, B. Tarasinski, D. Eeltink, V. S. Pribiag, S. R. Plissard, E. P. A. M. Bakkers, L. P. Kouwenhoven, and M. Wimmer. Spin-orbit interaction in insb nanowires. Physical Review B, 91(20):201413, 2015. 